\title{
Using Free Double Barrel Vascularized Fibular Grafting in Complex Nonunion with Extensive Bone Loss in Distal Humeral Fractures
}

\section{Saab Mufleh Al Mestirihi ${ }^{1 *}$, Yousef Marzouq Khair ${ }^{1}$, Ayman Mustafa Burghol $^{2}$, Sherif Mamdouh AMR ${ }^{3}$, Sana'a Haddadin ${ }^{4}$ and Silvana De Giorgi $^{5}$}

${ }^{1}$ King Hussein Medical Center, Royal Medical Services, Amman, Jordan

${ }^{2}$ MD Orthopedic Surgeon, Upper Limb Department, Royal Medical Services,

Amman, Jordan

${ }^{3}$ Professor, Orthopedic Consultant, Cairo University, Cairo, Egypt

${ }^{4} R N$, Private Hospital, Amman, Jordan

${ }^{5}$ Professor, Department of Basic Medical Sciences, Neurosciences and Sensory

Organs, University of Bari, Italy

*Corresponding Author: Saab Mufleh Al Mestirihi, Hand and Upper Limb

Department, Royal Medical Services, Amman, Jordan.

\author{
Received: May 03, 2021
}

Published: May 20, 2021

(C) All rights are reserved by Saab Mufleh Al

Mestirihi., et al.

\begin{abstract}
Distal humeral fractures are not very common. 3\% of these fractures may need further surgical management [22]. Nonunion of fractures of the distal humerus is a challenging issue for orthopedic surgeons. Management could be either by using non vascularized or vascularized bone grafts, or by using a double barrel vascularized graft from the fibula. In our study we used a double barrel vascularized fibular graft for treatment this kind of nonunion. We used this procedure with different ways of fixation (either using external fixation, $\mathrm{k}$ wires with screws, plates) in different patients. Double barrel vascularized fibular grafting is a good option for treatment of fracture nonunion with a gap of $6 \mathrm{~cm}$ or more. It adds stability to the medial and lateral columns and enhances bone healing at the fracture site.
\end{abstract}

Keywords: Double Barrel; Vascularized Fibular Graft; Non Union Humerus Fracture

\section{Introduction}

Fractures of the distal humerus represent $2 \%-3 \%$ of all fractures in adults; nonunion of fractures of the distal humerus is a challenging issue, its incidence amounting from $2 \%$ up to $10 \%$ $[12,13]$.

Seven patients in this study underwent open reduction internal fixation of the distal humerus with consequent metal failure and nonunion. All patients had bone defect of at least $5 \mathrm{~cm}$ with history of non-union fracture distal humerus for at least 2 years. They underwent subsequent revision surgery using double barrel vascularized fibular grafting stabilizing both columns, mostly the medial column, by plates, screws, or k wires. All patients had good results with satisfactory bone healing within 4 to 6 months after surgery.

Most of the studies are about using vascularized fibular graft in non-union distal humerus fracture, while there are rare studies about using double barrel vascularized fibular graft. Our study supports the technique using double barrel vascularized fibular graft, that is quite useful in cases with non-union distal humerus associ- 
ated with bone loss more than $5 \mathrm{~cm} \mathrm{[1,2].} \mathrm{We} \mathrm{tried} \mathrm{to} \mathrm{demonstrate}$ our hypothesis that using double barrel vasculariesd fibular graft based on brachial artery will give a predictable union and satisfactory results.

In the literature, some studies described free vascularized fibular graft [14]. Other studies described the benefits of using vascularized fibular graft in tumor reconstruction [15-17,20]. Other studies explained the benefits of using free vascularized fibular graft in bone defects $[18,19]$.

In our paper we used a double barrel vascularized fibular graft in management of non union distal humerus fracture with bone defect. We explain in this paper the biomechanical rationale behind using this technique and the surgical technique of performing this procedure.

\section{Methods}

This study was performed in co-operation between Cairo University Hospital in Cairo-Egypt and Royal medical services Amman-Jordan in the period between August 2015 to December 2018. All patients had nonunion after fractures of the distal humerus. These fractures had been managed primarily by external fixation or plating (Table 1). The patients presented with fracture nonunion 9 months after the first surgery either external fixation or plating (Figure 1). All of these fractures underwent revision by using double barrel vascularized fibular grafting fixed by screws; the mean follow up period was 21 months.

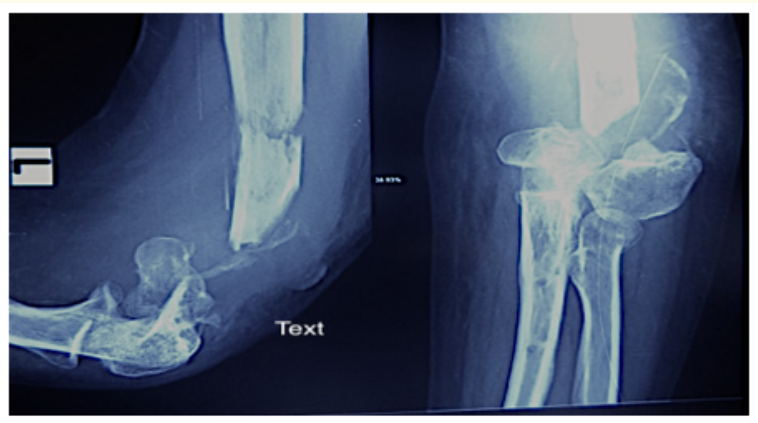

Figure 1: Illustrated lateral and AP views $x$-ray show non-union fracture distal humerus with bone loss.

The study group consisted of 7 patients Four males and three females as shown in table 1 . The mean age was 38 years, four with dominant arm and three with non-dominant arm. underwent open reduction internal fixation of the distal humerus with consequent metal failure and nonunion. All patients had bone defect of at least $5 \mathrm{~cm}$ with history of non-union fracture distal humerus for at least 9 months with no history of neurovascular defect. They underwent subsequent revision surgery using double barrel vascularized fibular grafting stabilizing both columns, mostly the medial column, by plates, screws, or $\mathrm{k}$ wires. In some cases we used bone cement as an adjunct to internal fixation and to increase the stability of the fixation that aid healing in most of the cases. Bone cement had the characteristics of improving bone rigidity and strength, and through applying bone cement strengthening measures, it could effectively reduce the pain caused by bone deformation and the micro stimulation of fracture site; More heat and free radicals were formed during bone cement polymerization, which destroyed sensitive nerve endings in the stroma and helped relieve pain; in our cases the bone cement was in the other site from the site of fixation in the fracture non-union fracture and the purpose of obtaining the filling of the bone cement into the fracture was achieved, thereby enhancing the stability of the fracture [23].

\begin{tabular}{|l|c|c|c|c|}
\hline Age & Sex & $\begin{array}{c}\text { Right arm or } \\
\text { left arm }\end{array}$ & Type of defect & $\begin{array}{c}\text { Previous } \\
\text { surgery }\end{array}$ \\
\hline 40 & M & Right arm & Loss of bone & External fixation \\
\hline 35 & M & Right arm & $\begin{array}{c}\text { Distal humerus } \\
\text { fracture }\end{array}$ & Plate \\
\hline 44 & F & Right arm & $\begin{array}{c}\text { Fracture distal } \\
\text { humerus extended to } \\
\text { intraarticular area }\end{array}$ & Plate \\
\hline 33 & M & Left arm & $\begin{array}{c}\text { Comminuted fracture } \\
\text { distal humerus }\end{array}$ & Plate \\
\hline 38 & F & Left arm & $\begin{array}{c}\text { Distal humerus } \\
\text { fracture }\end{array}$ & Plate \\
\hline 42 & M & Right arm & $\begin{array}{c}\text { Comminuted fracture } \\
\text { distal humerus }\end{array}$ & External fixation \\
\hline 23 & F & Left arm & $\begin{array}{c}\text { Comminuted fractur } \\
\text { distal humerus }\end{array}$ & External fixation, \\
cement spacer
\end{tabular}

Table 1: Demographic data of the patients.

We followed up the patients and X-rays were used for assessment of the healing process of the bone. we used Linscheid and Wheeler grading system which considers results, number of the patients and the criteria (Table 2).

\section{Results}

In all patients, the union of the fracture was obtained within a mean interval of 5 months. We classified the patients according 
to Linscheid and Wheeler grading system. Five patients had less than 15 degree extension or flexion with no pain or instability, two patients had less than 30 degree extension or flexion, pronation or supination loss, mild complain of instability or pain on heavy use. There was improvement in range of motion in all patients (Table 2).

\begin{tabular}{|l|c|c|}
\hline \multicolumn{1}{|c|}{ Result } & Patients (n) & Criteria \\
\hline Excellent & 0 & Full range of motion, normal elbow \\
\hline Good & 5 & $\begin{array}{c}<15^{\circ} \text { extension or flexion loss, no } \\
\text { pain or instability. }\end{array}$ \\
\hline Fair & 2 & $\begin{array}{c}<30^{\circ} \text { extension or flexion, pronation } \\
\text { or supination loss, mild complain of } \\
\text { instability or pain on heavy use. }\end{array}$ \\
\hline Poor & 0 & $\begin{array}{c}\geq 30^{\circ} \text { extension or flexion, pronation } \\
\text { or supination loss, residual pain, } \\
\text { instability of neurovascular } \\
\text { disability. }\end{array}$ \\
\hline
\end{tabular}

Table 2: Results graded according to Linscheid and Wheeler.

The distal humerus fracture was classified according AO classification as group A in two patients, as group B in three patients and as group $\mathrm{C}$ in one patient [21]. Anteroposterior and lateral plain radiographic views were used after surgery and during follow up to assess the healing process and union of the bone.

All patients had good results with significant bone healing within 4 to 6 months after surgery, with free pain functional range of motion.

\section{Discussion}

In this study, although the number of patients was limited, all of them are included in the study and had satisfactory results. According to the grading evaluation we considered the Linscheid and Wheeler grading system (Table 2). The bone healed in a period between 4 to 6 months, with no sever pain and good range of motion.

This study lended support to other studies about the benefits of using double barrel vascularized fibular grafting [1-4]. There is another study which described the use of fibular graft in tumor reconstruction [5-7]; other studies explained the benefits of using free vascularized fibular graft in bone defects [8-10].

Ackerman and Jupiter [11] reported on 20 patients with nonunion of fracture of the distal humerus. The original fracture was classified according to AO classification, nine patients were group A2, four group A3, one group C1, three group C2 and three group C3.
Sanders and Sackett [12] reported on five patients; two were group C1, one was group C2, one group C3 and one group D1 (an intraarticular fracture of the capitellum and the trochlea in transverse plane). It was proved that normal union bone could be achieved within few months by using this kind of graft; factors enhancing union were deemed unnecessary.

Selecting the correct implant with appropriate placement of the implant, and the biological healing potential of the bone in order to encourage union as described by Saunders and Sackett [12] are important concepts.

The mechanism of injury didn't appear to be significant as most of the patient had history of simple trauma. The largest series of nonunion is that by Helfet., et al. [12] who described the management of 52 patients; they were able to classify the configuration of the nonunion, but did not record the original pattern of fracture.

One important factor leading to fracture nonunion is the type and method of management after injury. After restudying the radiographs of the patients and examining them we considered that they had not had adequate management for the primary fracture.

Posterior humeral approach was used for preparing the site of nonunion. Tourniquet use was abandoned. The ulnar nerve was identified and protected by tape after having been decompressed. The triceps was either split or released medially and laterally. The fracture site at the distal humerus was explored, the nonunion was debrided, the fibrous tissue and dead bone fragments were excised. Through an anterior humeral utilitarian approach, the brachial vessels and median nerve were exposed. The fracture site was exposed anteriorly. Then the vascularized fibular graft was extracted via a lateral approach according to the technique described by other authors. The double barrel fibular graft was prepared as described elsewhere taking care not to injury the pedicle (Figure $2-5)$. The vascularized fibular graft was fixed to the medial aspect of the distal humerus in a double barrel fashion using 3.5 screws (Figure 6a). The peroneal vessels of the fibular pedicle were anastomosed to the brachial vessels in an end-to-side fashion. After reestablishment of flow into the peroneal vessels, the vascularity of the graft was checked. The elbow was protected in 90 degrees by a back slab for three weeks. After that, gentle physiotherapy was started.

Most of the studies explain using vascularized fibular graft in non union distal humerus fracture and there are rare studies that 


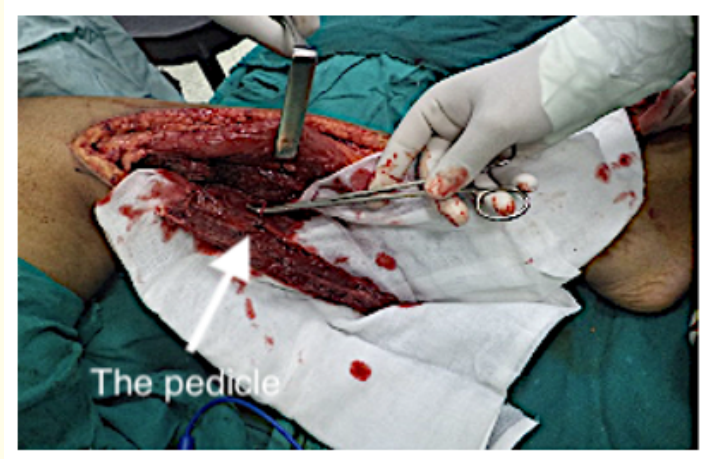

Figure 2: Illustrated a lateral approach for the leg with longitudinal cut of fibula.

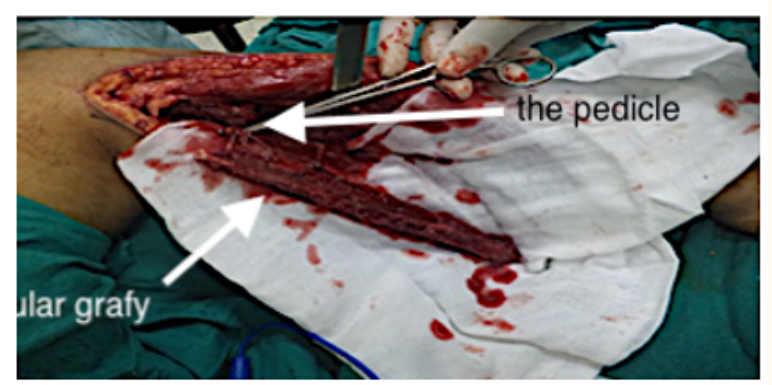

Figure 3: Illustrated dissection around the fibula with preserving the pedicle.

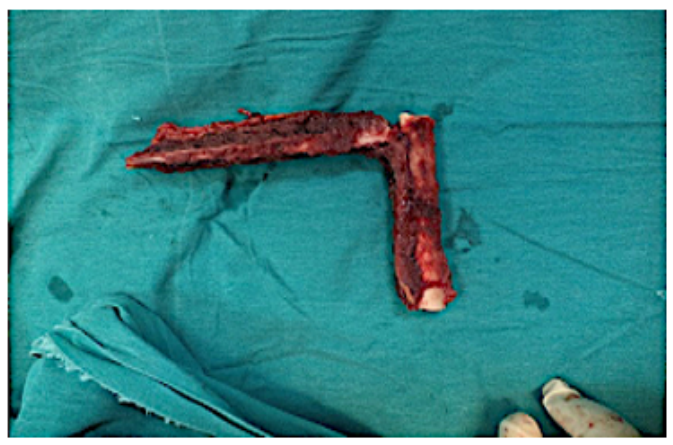

Figure 4: Illustrated divided the fibular graft into two parts.

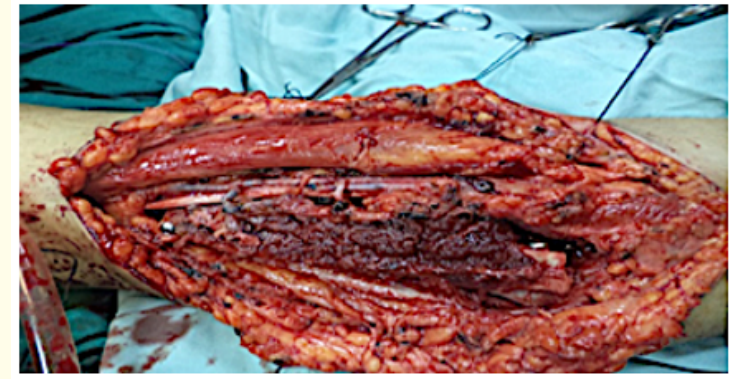

Figure 5: Illustrated a vascularized graft is fixed in distal humerus.

discussed using double barrel vascularized fibular graft. Our study supports the studies of the technique using double barrel vascularized fibular graft is useful in cases with non union distal humerus associated with bone loss more than $5 \mathrm{~cm}$, we tried to corroborate our hypothesis that using double barrel vasculariesd fibular graft based on brachial artery will give a predictable union and satisfactory results (Figure 6b).

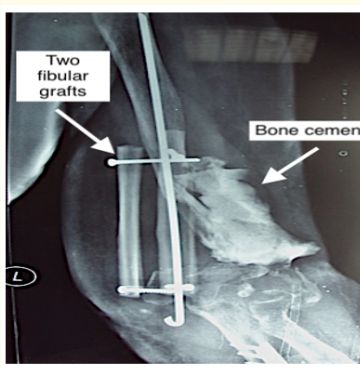

a

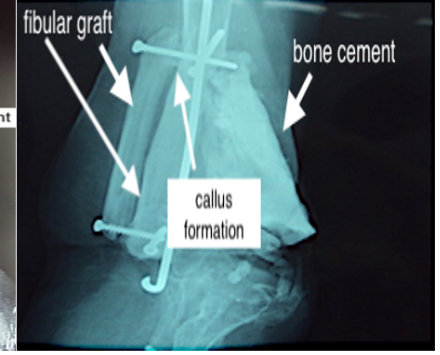

b
Figure 6a and 6b: a: The arrows show the two fibular graft which one screw fixed by the graft and the proximal part of the non union site vertically and one screw with distal part of the graft with distal humerus and on the other site the bone cement. b: For the same patients which shows callus formation at the graft site.

\section{Conclusion}

Fractures at the intercondylar and supracondylar regions are close to the elbow joint. They usually cause stiffness to the elbow joint, so the motion is transmitted to the fracture site. This leads to 
bone resorption at the fracture site. Thus, the joint has to be arthrolyzed to promote bone union at the fracture site.

Double barrel grafting has the advantage of fixing both medial and lateral columns of the distal humerus. Thus, union occurs over a large surface, enhance stability mostly on the medial column. Using double barrel vascularized fibular graft based on brachial artery can give a predictable union and satisfactory results.

\section{Competing Interests}

The authors declare no competing interest.

\section{Bibliography}

1. Jones NF., et al. "The "double barrel" free vascularized fibular bone graft". Plastic and Reconstructive Surgery 81.3 (1988): 378-385.

2. Banic A and Hertel R. "Double vascularized fibulas for reconstruction of large tibial defects". Journal of Reconstructive Microsurgery 9.6 (1993): 421-428.

3. Bähr W., et al. "Use of the "double barrel" free vascularized fibula in mandibular reconstruction". Journal of Oral and Maxillofacial Surgery 56.1 (1998): 38-44.

4. Guerra MF., et al. "The partial double-barrel free vascularized fibular graft: a solution for long mandibular defects". Plastic and Reconstructive Surgery 105.5 (2000): 1902-1903.

5. Amr SM., et al. "Reconstruction after resection of tumors around the knee: role of the free vascularized fibular graft". Microsurgery 20.5 (2000): 233-251.

6. El-Gammal TA., et al. "Microsurgical reconstruction of lower limb bone defects following tumor resection using vascularized fibula osteoseptocutaneous flap". Microsurgery 22.5 (2002): 193-198.

7. El-Gammal TA., et al. "Reconstruction of lower limb bone defects after sarcoma resection in children and adolescents using free vascularized fibular transfer". Journal of Pediatric Orthopaedics B 12.4 (2003): 233-243.

8. Bi ZG., et al. "Reconstruction of large limb bone defects with a double-barrel free vascularized fibular graft". Chinese Medical Journal 121.23 (2008): 2424-2428.

9. Chu CH., et al. "Reconstruction of a massive femoral bone defect using a double-barreled free vascularized fibular bone graft after wide resection of femoral chondrosarcoma". The Kaohsiung Journal of Medical Sciences 25.10 (2009): 552-558.

10. Tanaka K., et al. "Vascularized fibular graft for bone defects after wide resection of musculoskeletal tumors". The Journal of Orthopaedic Science 17.2 (2012): 156-162.

11. Wieser K., et al. "Autologous double-barrel vascularized fibula bone graft for arthrodesis of the shoulder after tumor resection". Archives of Orthopaedic and Trauma Surgery 133.9 (2013): 1219-1224.

12. A Ali., et al. "Revision surgery for nonunion after early failure of fixationof fractures of the distal humerus". Journal of Bone and Joint Surgery 87 (2005).

13. Ackerman $G$ and Jupiter JB. "Non-union of fractures of the distal end of the humerus". Journal of Bone and Joint Surgery American 70 (1988): 75-83.

14. Marko Bumbasirevic, Milan Stevanovic, Vesna Bumbasirevic, Aleksandar Lesic and Henry D.E.Atkinson (2014).

15. Barnds B., et al. "Ipsilateral rotational double-barrel fibula autograft for limb salvage in a pediatric patient with lower extremity intramedullary osteosarcoma: A case report”. Microsurgery (2019).

16. Wieser K., et al. "Autologous double-barrel vascularized fibula bone graft for arthrodesis of the shoulder after tumor resection". Archives of Orthopaedic and Trauma Surgery 133.9 (2013): 1219-1224.

17. Chu CH., et al. "Reconstruction of a massive femoral bone defect using a double-barreled free vascularized fibular bone graft after wide resection of femoral chondrosarcoma". The Kaohsiung Journal of Medical Sciences 25.10 (2009): 552-558.

18. Cano-Luís P., et al. "Treatment of posttraumatic bone defects of the forearm with vascularized fibular grafts. Follow up after fourteen years". Injury 49.2 (2018): S27-S35.

19. Bi ZG., et al. "Reconstruction of large limb bone defects with a double-barrel free vascularized fibular graft". Chinese Medical Journal 121.23 (2008): 2424-2428.

20. Amr SM., et al. "Reconstruction after resection of tumors around the knee: role of the free vascularized fibular graft". Microsurgery 20.5 (2000): 233-251. 
21. Beeres FJ., et al. "[Distal humerus fracture-extensile approaches]". Operative Orthopädie und Traumatologie 29.2 (2017): $115-124$

22. Robinson CM., et al. "Adult distal humeral metaphyseal fractures: epidemiology and results of treatment". Journal of Orthopaedic Trauma 17.1 (2003): 38-47.

23. Zou H., et al. "Augmentation of pedicle screw fixation with percutaneous vertebroplasty with bone cement in treatment of senile osteoporotic vertebral compression fractures associated with spinal stenosis". Journal Neck Pain 24 (2015): 93-99.

\section{Volume 4 Issue 6 June 2021}

(C) All rights are reserved by Saab Mufleh Al Mestirihi., et al. 